LCLS-TN-05-04

\title{
Earth’s Magnetic Field Measurements for the LCLS Undulators
}

\author{
Kirsten Hacker, Zachary Wolf \\ SLAC
}

February 16, 2005

\begin{abstract}
Measurements of the earth's magnetic field at several locations at SLAC were conducted to determine the possible field error contribution from tuning the undulators in a location with a different magnetic field than that which will be found in the undulator hall. An average difference of $0.08+$ 0.04 Gauss has been measured between the downward earth's field components in the test facility and SLAC tunnel locations.
\end{abstract}

\section{Introduction}

There are concerns that the difference between the magnetic field in the laboratory where the undulators will be tuned and the magnetic field in the undulator hall where they will be used is large enough to affect the performance of LCLS. The earth's magnetic field, in general, is large enough to affect the performance of LCLS and for that reason the undulators will be measured and tuned in the same orientation in which they will be installed in the undulator hall. This technique of tuning out the earth's magnetic field will be effective if the field in the laboratory is the same as it is in the undulator hall. This report contains a preliminary investigation into the validity of this assumption. Since the undulator hall and magnetic measurements laboratory are not yet constructed, measurements were performed inside and outside of various buildings at several locations on the SLAC site.

The average difference between the earth's downward magnetic field component outside of the undulator magnetic measurements facility and the field at the location of the undulator hall construction site is $-0.01 \pm 0.01$ Gauss. The average difference between the downward field component in the room that will house the undulator test bench and four different SLAC tunnel locations is $0.08 \pm 0.04$ Gauss. Although, there are many large metal objects within the tunnels and in the undulator test facility building that affect the ambient magnetic field components by more than 0.95 Gauss, there are areas with a downward magnetic field component constant to better than 0.01 Gauss. Thus, local magnetic objects add uncertainty when extrapolating to a new lab and a new tunnel. 


\section{Measurement method}

The device used to measure the earth's magnetic field was the Mag-03PSU, consisting of 3 orthogonally mounted fluxgate sensing elements with less than 0.05 milliGauss measurement error. The $\mathrm{z}$ direction was lined up with magnetic north with a compass and the y direction was pointed downwards and aligned with a level (Fig. 1).

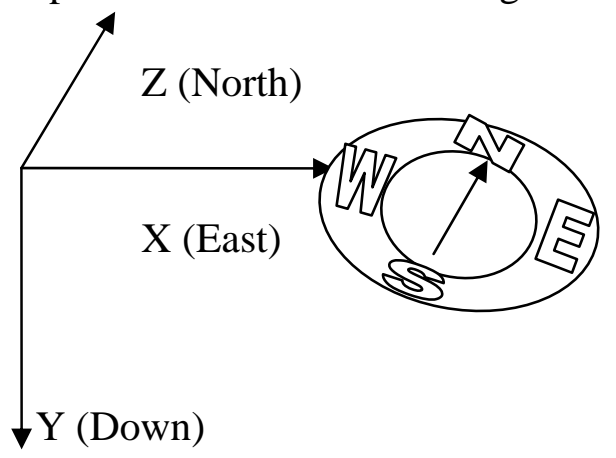

FIG 1. Field measurement coordinates. North corresponds to Z, East is X, and Down is Y. A compass was used for alignment in $\mathrm{X}$ and $\mathrm{Z}$. A level was used to adjust the $\mathrm{Y}$ alignment.

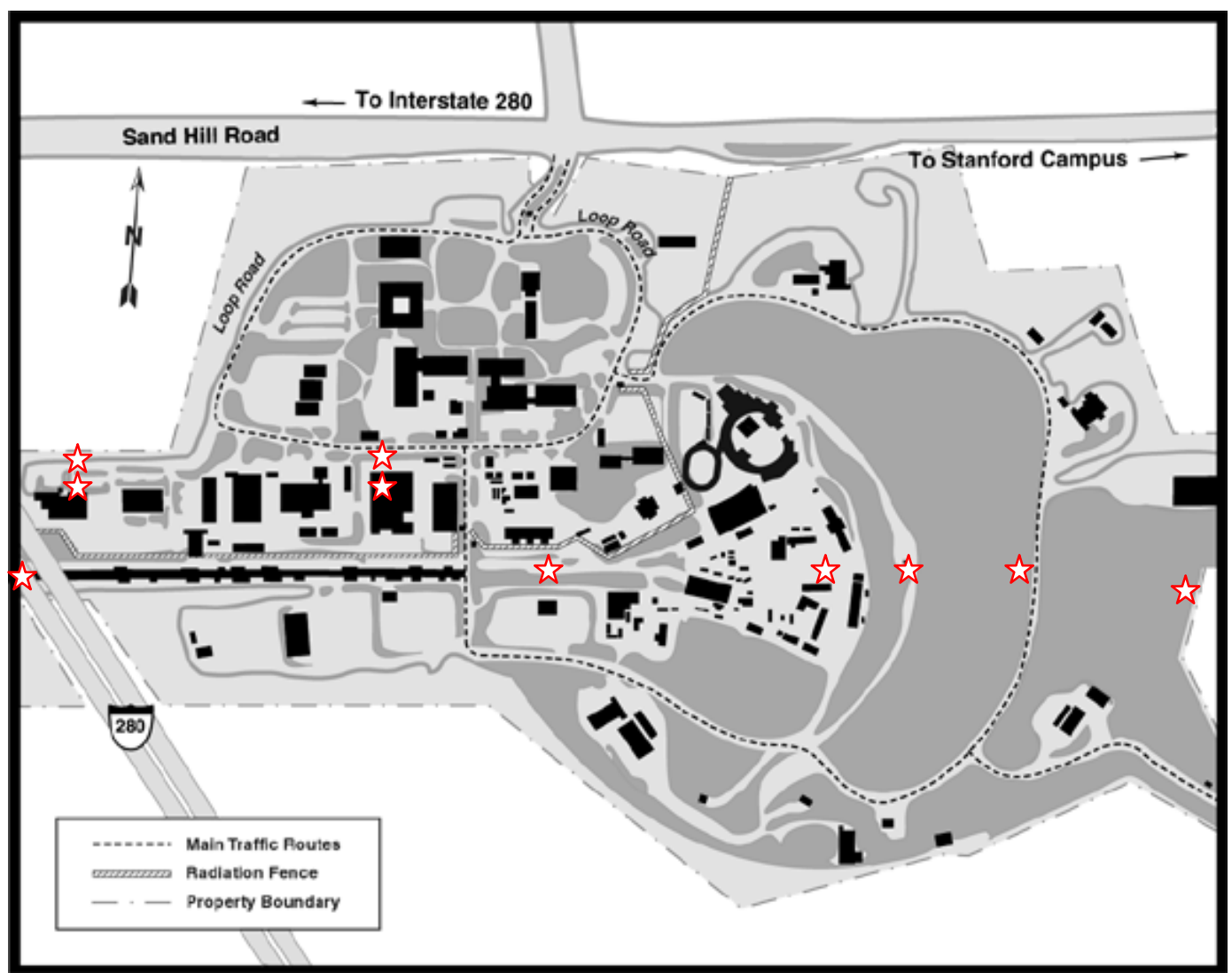

FIG 2. SLAC site map with measurement locations. Measurements of the earth's magnetic field were performed inside and outside of the present lab and the new lab location as well as in the FFTB, linac sector 20, the south SLC arc, the research yard, and the PEP loop road. 
The measurement sites, represented by stars in Fig. 2, were chosen to compare the likely inside and outside field components of the undulator test facility and the undulator hall. Measurements were made inside and outside of the new lab location (building 81), the present lab (building 26), the FFTB, linac sector 20, SLC south arc, and the outdoor undulator hall building site (access road and PEP loop road).

At each building site measurements were completed in four locations, separated by 1 to 4 meters. The inside locations are full of metal objects that make it difficult to find large areas with constant ambient magnetic fields. Efforts were made to make the measurements in the most metal-free regions available. The field measurements in building 81, the location of the future undulator test facility, were done at waist height around the expected position of the test bench with visible metal objects moved at least 2.5 meters away. The measurements in the FFTB were done on the floor in a relatively empty space between CB01 5030 and CB01 5020. SLC south arc measurements were done at 1 meter height in the center of the walkway in both the spray on concrete section and the concrete slab section. The spray on concrete is infused with small metal flecks and the concrete slab is supported by rebar. The linac measurements were done at 1.5 meter height in sector 20, a region with accelerating structures and no magnets.

Magnetic north, chosen here as the $Z_{\text {mag }}$ direction, is 64 degrees $(1.053 \mathrm{rad})$ from the beam direction in the linac. The easterly beam direction is the $Z_{\text {linac }}$ coordinate, $X_{\text {linac }}$ points in the northerly direction, and $\mathrm{Y}_{\text {linac }}$ points up. So a transformation from the magnetic to linac coordinates would look like:

$$
\left(\begin{array}{ccc}
-0.4382 & 0 & 0.8989 \\
0 & -1 & 0 \\
0.8989 & 0 & 0.4382
\end{array}\right)\left(\begin{array}{l}
B_{X_{\text {mag }}} \\
B_{Y_{\text {mag }}} \\
B_{Z_{\text {mag }}}
\end{array}\right)=\left(\begin{array}{l}
B_{X_{\text {linac }}} \\
B_{Y_{\text {linac }}} \\
B_{Z_{\text {linac }}}
\end{array}\right)
$$

The results presented in the following section are given in the magnetic coordinate system and not the linac coordinate system.

Since the variation of the measurement is dominated, not by measurement device error, but by alignment error and field disturbances caused by building materials, the standard deviation of several measurements done at different locations will be given as the error. The $\mathrm{X}$ and $\mathrm{Z}$ alignment error is due primarily to local field disturbances and in the linac it was \pm 20 degrees, as measured with the compass. This means that, although $X$ is always aligned so that it is very close to zero, $\mathrm{Z}$ may vary by $7 \%$ relative to a fixed coordinate system, due to misalignment coupling with the $\mathrm{X}$ coordinate.

\section{Results}

The average field magnitude at the future location of the undulator test bench is $0.41 \pm$ 0.02 Gauss. In the tunnel it is $0.43 \pm 0.06$ Gauss and outside it is $0.49 \pm 0.01$ Gauss (Fig. 4). The larger standard deviation of the indoor samples is due to the close proximity of metal objects and the smaller magnitude is due to the shielding effects of building materials. Indoor and outdoor samples and averages are presented in Fig.s 3 and 4. Examples of field changes due to large metal objects are presented in Fig. 5 and the 
indoor differences between the planned undulator test facility and SLAC tunnel field measurements are presented in Fig. 6.

\begin{tabular}{|l|c|l|l|l|}
\hline Inside & X (Gauss) & Y (Gauss) & Z (Gauss) & Magnitude \\
\hline Bldg 81 & $0.01 \pm 0.01$ & $0.31 \pm 0.03$ & $0.27 \pm 0.04$ & $0.41 \pm 0.02$ \\
\hline Bldg 26 & $-0.02 \pm 0.04$ & $0.18 \pm 0.10$ & $0.24 \pm 0.07$ & $0.32 \pm 0.02$ \\
\hline SLC arc spray & $0.02 \pm 0.02$ & $0.43 \pm 0.01$ & $0.24 \pm 0.01$ & $0.50 \pm 0.01$ \\
\hline SLC arc slab & $0.00 \pm 0.01$ & $0.37 \pm 0.01$ & $0.24 \pm 0.01$ & $0.44 \pm 0.01$ \\
\hline Sect 20 & $0.00 \pm 0.01$ & $0.33 \pm 0.07$ & $0.12 \pm 0.02$ & $0.35 \pm 0.06$ \\
\hline FFTB & $-0.06 \pm 0.02$ & $0.40 \pm 0.08$ & $0.16 \pm 0.06$ & $0.44 \pm 0.07$ \\
\hline
\end{tabular}

\begin{tabular}{|l|l|l|l|l|}
\hline Outside & X (Gauss) & Y (Gauss) & Z (Gauss) & Magnitude \\
\hline Bldg 26 & $0.00 \pm 0.02$ & $0.41 \pm 0.02$ & $0.27 \pm 0.02$ & $0.48 \pm 0.04$ \\
\hline Bldg 81 & $0.02 \pm 0.01$ & $0.42 \pm 0.01$ & $0.25 \pm 0.01$ & $0.49 \pm 0.01$ \\
\hline Access road & $0.00 \pm 0.02$ & $0.42 \pm 0.01$ & $0.24 \pm 0.01$ & $0.49 \pm 0.01$ \\
\hline PEP road & $0.00 \pm 0.02$ & $0.43 \pm 0.01$ & $0.24 \pm 0.01$ & $0.49 \pm 0.01$ \\
\hline Research yard & $0.00 \pm 0.01$ & $0.42 \pm 0.01$ & $0.24 \pm 0.01$ & $0.48 \pm 0.01$ \\
\hline
\end{tabular}

FIG 3. Earth's magnetic field measurements. Measurements of the earth's magnetic field were performed inside and outside of several locations. The error represents the standard deviation over 4 measurements. Building 26 is the current magnetic measurements facility and building 81 is the site of the future undulator measurement facility. SLC arc spray and slab samples were taken in regions of the arcs with different building materials

\begin{tabular}{|l|l|l|l|l|}
\hline Location & X (Gauss) & Y (Gauss) & Z (Gauss) & Magnitude \\
\hline Average bldg 81 & $-0.02 \pm 0.04$ & $0.31 \pm 0.03$ & $0.27 \pm 0.04$ & $0.41 \pm 0.02$ \\
\hline Average tunnel & $-0.01 \pm 0.03$ & $0.38 \pm 0.07$ & $0.20 \pm 0.05$ & $0.44 \pm 0.06$ \\
\hline Average outside & $0.00 \pm 0.02$ & $0.42 \pm 0.01$ & $0.25 \pm 0.02$ & $0.49 \pm 0.01$ \\
\hline
\end{tabular}

FIG 4. Earth's field inside and outside of lab and tunnels. Building materials make the magnetic field more variable and weaker.

\begin{tabular}{|l|l|l|l|l|}
\hline FFTB Location & Distance (m) & X (Gauss) & Y (Gauss) & Z (Gauss) \\
\hline Quadrupole & .5 & 0.20 & 0.17 & 0.02 \\
\hline Steel girder & .25 & 0.95 & 0.20 & 0.36 \\
\hline Nominal field & 1.5 & -0.06 & 0.40 & 0.16 \\
\hline
\end{tabular}

FIG 5. FFTB de-energized magnetic-field measurements of objects that perturb the field locally. 


\begin{tabular}{|l|l|l|l|c|}
\hline$\Delta$ Location & $\boldsymbol{\Delta} \mathbf{X}$ (Gauss) & $\boldsymbol{\Delta} \mathbf{Y}$ (Gauss) & $\boldsymbol{\Delta} \mathbf{Z}$ (Gauss) & $\Delta$ Magnitude \\
\hline FFTB - lab & $-0.07 \pm 0.02$ & $0.09 \pm 0.08$ & $-0.11 \pm 0.06$ & $0.03 \pm 0.07$ \\
\hline SLCslab - lab & $-0.03 \pm 0.01$ & $0.08 \pm 0.03$ & $-0.04 \pm 0.04$ & $0.05 \pm 0.02$ \\
\hline SLCspray - lab & $-0.01 \pm 0.02$ & $0.13 \pm 0.03$ & $-0.04 \pm 0.04$ & $0.08 \pm 0.02$ \\
\hline Linac - lab & $-0.01 \pm 0.02$ & $0.02 \pm 0.07$ & $-0.15 \pm 0.04$ & $-0.06 \pm 0.06$ \\
\hline
\end{tabular}

\begin{tabular}{|l|l|l|l|l|}
\hline Average & $-0.02 \pm 0.04$ & $0.08 \pm 0.04$ & $-0.08 \pm 0.06$ & $-0.02 \pm 0.06$ \\
\hline
\end{tabular}

FIG 6. The average field inside the tunnel locations minus the average field at the location of the undulator test bench and the average over the four locations.

In locations away from large structures, the downward component of the earth's magnetic field outside of buildings varies across the SLAC site by 0.01 Gauss. Building materials such as the corrugated steel of the future undulator test facility and the steel reinforced concrete of the SLAC tunnels, however, attenuate the earth's magnetic field by different amounts. The difference between the downward field component in the future location of the undulator test bench and the average over several SLAC tunnel locations is $0.08 \pm 0.05$ Gauss. Some of the error of this measurement is contributed by field variations across the space to be occupied by the test bench, and this will be reduced when the steel racks presently occupying the site are removed.

\section{Conclusion}

Measurements of the earth's magnetic field at several locations at SLAC were conducted to determine the possible field error contribution from tuning the undulators in a location with a different magnetic field than that which will be found in the undulator hall. An average difference of $0.08 \pm 0.05$ Gauss has been measured between the downward earth's field components in the test facility and SLAC tunnel locations. 